\title{
Recurrent Severe Aplastic Anemia
}

National Cancer Institute

\section{Source}

National Cancer Institute. Recurrent Severe Aplastic Anemia. NCI Thesaurus. Code C153294.

The reemergence of severe aplastic anemia after a period of remission. 